\title{
Le passage à TARMED en 7 points
}

G. Claude

Les assureurs, les politiciens, les médecins voient tous dans l'introduction de TARMED des défis et des réponses qui vont dans le sens de leurs préoccupations, de leurs motivations ou de leurs craintes. Si certains plaident pour la grande révolution, d'autres y voient simplement «un changement de tarif». Comme dans toute évolution, des opportunités et des ruptures se créent. Après l'introduction de TARMED, les délégués aux négociations vont, dans le cadre de la neutralité des coûts, défendre les intérêts de chacune des parties. Si le niveau du point va effectivement modifier les recettes des médecins, certains autres aspects ne vont pas être du ressort des délégués aux négociations et devront être gérés par chacun des médecins. Voici une liste non exhaustive des 7 points à maîtriser pour gérer au mieux le passage à TARMED.

\section{Reconnaissance des droits acquis}

Après avoir obtenu la reconnaissance des droits acquis, le médecin connaît la liste des prestations qu'il peut facturer sous TARMED. La reconnaissance des droits acquis (concept de la valeur intrinsèque) est primordiale puisque c'est à ce stade que le médecin détermine l'étendue des prestations qu'il pourra facturer. Un «oubli» peut s'avérer fâcheux à long terme.

\section{Adaptation de votre logiciel de facturation}

- Le fournisseur de votre logiciel a-t-il intégré l'entier du tarif dans sa base de données? Pour éviter des erreurs, est-il possible d'indiquer les prestations que vous pouvez facturer (valeurs intrinsèques)?

- Est-ce que le logiciel gère les prestations additionnelles que vous pouvez facturer à une prestation de base?

Correspondence:

FMH Services Fiduciaire Monsieur Gérald Claude Av. des Prés Beudin 20

CH-1920 Martigny

Tél. 0277232009

Fax 0277232001

E-mail:

gerald.claude@fmhfiduciaire.ch
- Gère-t-il les possibilités de majoration (p. ex. pour les cas d'urgence ou de fractures ouvertes)?

- Est-il possible de facturer selon d'autres tarifs? Par exemple, la liste des spécialités (LIMA) ou encore le CPH?

\section{Facturation électronique et centre de confiance}

A terme, le grand changement impliqué par TARMED sera le mode de facturation. Jusqu'à présent, les factures étaient le plus souvent échangées sur un support papier. Il incombait alors aux caisses maladie de les saisir manuellement dans leur système informatique. Avec TARMED, nous devrions passer plus ou moins rapidement à une facturation électronique évitant la «re-saisie» par les caisses maladie. Afin de disposer d'une base statistique dans le cadre des négociations futures liées à la neutralité des coûts, les sociétés médicales cantonales ont créé les centres de confiance. Ainsi, les caisses maladie auront accès aux informations par le biais des ordinateurs des centres de confiance, lesquels transmettront les fichiers transmis par les médecins. Dans ce contexte, vous devez réfléchir à l'adhésion à un centre de confiance et vous assurer que votre fournisseur de logiciels puisse fournir les fichiers selon les standards admis.

\section{Utilisation de TARMED}

Si les anciens tarifs cantonaux fonctionnaient en «idéogrammes», nous passons à un tarif en lettres où tout est découpé et détaillé. Cette nouvelle façon de facturer est effectivement à apprivoiser et la façon de l'appliquer est un apprentissage important pour lequel il convient de s'appuyer sur le «Validator». En effet, de nombreuses prestations sont limitées dans un temps donné ou ne peuvent pas être facturées avec d'autres. Ces tests sont nombreux et seront effectués avant paiement par les caisses maladies. De même, il est nécessaire de garder à l'esprit que TARMED n'est qu'un des tarifs à appliquer. Par exemple, concernant le matériel, la liste des spécialités pour le matériel et les accessoires (LIMA) reste utilisable. Par exemple, pour le matériel de 
moins de Fr. 3.-, cette liste peut s'avérer être d'une aide précieuse. De même, la liste OFAS pour des examens de laboratoire ou le CPH (ou d'autres tarifs privés) pour des interventions chirurgicales en cas d'hospitalisations privées sont à utiliser.

\section{Points particuliers à surveiller}

\section{Liquidités}

L'adaptation des systèmes informatiques (médecin, centre de confiance et caisses maladie) va prendre du temps. Il en est de même de l'apprentissage du tarif par les employés des cabinets médicaux et des caisses maladies. Les tests effectués à l'aide du «Validator» sont nombreux et seront effectués avant le paiement par les caisses maladies. Des prestations non admises entre elles, des dépassements de limitation ou encore des prestations non admises selon l'inventaire de vos droits acquis vont être source de refus de prise en charge par les caisses maladie. Elles pourront alors, soit rembourser partiellement la facture, soit en refuser le paiement intégral. Si l'on considère que la grande majorité des patients paient leur médecin après avoir encaissé la prise en charge de la caisse maladie, on peut s'attendre à un «décalage» dans le temps du remboursement des prestations et par conséquent du paiement des honoraires aux médecins. Ainsi, il est tout à fait probable de voir des factures «en suspens». Il en résultera une période creuse dans l'encaissement des prestations par les médecins.

\section{Structure du cabinet}

TARMED va influencer le niveau des recettes de certains cabinets. Dès lors, il est impératif d'étudier l'adéquation de la structure du cabinet et d'envisager des solutions d'adaptation (sous-traitance de prestations, réduction des frais fixes ...).

\section{Fiscalité}

Dans le cas où il existe un retard dans la facturation des prestations (existence de réserves latentes lors du dernier bouclement comptable), il convient d'anticiper le fait que toutes les prestations devront être facturées au 31 décembre 2003. Par conséquent, le bénéfice du cabinet de 2003 va être plus important. Pour éviter une charge fiscale plus importante, il convient d'analyser si d'autres mesures peuvent être prises (p. ex. rachat à la LPP, frais d'entretien de bâtiment ...).

\section{Contrôle des prestations}

TARMED permettra aux caisses maladie de disposer d'informations beaucoup plus conséquentes et détaillées que jusqu'à présent. La reconstitution des chaînes de traitement (le prescripteur étant saisi par son code EAN), la saisie de code de diagnostic (ICD-10) ou tout simplement le fait de facturer en minutes permettront des contrôles beaucoup plus importants. Il convient d'intégrer cette nouvelle conception du contrôle pour éviter que de simples erreurs de saisie ou d'utilisation du tarif ne soient pas mal interprétées.

\section{Neutralité des coûts et évolution future}

L'évolution future de la valeur du point ou les adaptations de TARMED vont nécessiter de nouvelles adaptations en fonction de la situation de chacun (p. ex. hausse ou baisse de la valeur du point selon le canton). La concurrence entre les prestataires de différents cantons ou les centres de confiance va peut-être influencer la réalité de demain! L'avenir nous montrera l'évolution de la situation.

\section{Ihr Partner, wenn Zahlungs- eingänge sich verzögern!}

Säumige Patientinnen und Patienten? Wenn die Zahlungsmoral krankt, ist Erfahrung gefragt. Wir sind die Spezialisten, entlasten Sie von Inkassosorgen und treiben für Sie die Honorarforderungen ein.

Und all dies zu fairen Konditionen, mit grosser Sensibilität und mit der gefragten Kompetenz. Damit Sie sich aufs Wesentliche konzentrieren können. Zum Wohl Ihrer Patientinnen und Patienten.

\section{Votre partenaire pour le règle- ment de vos factures!}

Avez-vous des patients mauvais payeurs? Si cela vous irrite, faites appel à notre expérience. Nous sommes des spécialistes, nous nous occupons de vos soucis de contentieux et encaissons pour vous les honoraires dus!

Et tout ceci à des conditions équitables, avec tact et compétence. Pour que vous puissiez vous concentrer sur l'essentiel. Pour le bien des vos patients et patientes.
FMH Inkasso Services,

Thorackerstrasse 3, 3074 Muri b. Bern

Tel. 03195080 30, Fax 03195080 40,

E-Mail: mail@fmhinkasso.ch

\section{www.FMHjob.ch}

Die Stellenplattform für Ärzte/Office de Placement online.

Bestandteil des Dienstleistungsangebotes der FMH Consulting Services 